\title{
An MRI study of temporal lobe abnormalities and negative symptoms in chronic schizophrenia
}

\section{Citation}

ANDERSON, J, C WIBLE, R MCCARLEY, M JAKAB, K KASAI, and M SHENTON. 2002. An MRI Study of Temporal Lobe Abnormalities and Negative Symptoms in Chronic Schizophrenia. Schizophrenia Research 58, no. 2-3: 123-134. doi:10.1016/s0920-9964(01)00372-3. http:// dx.doi.org/10.1016/s0920-9964(01)00372-3.

\section{Published Version}

doi:10.1016/s0920-9964(01)00372-3

\section{Permanent link}

http://nrs.harvard.edu/urn-3:HUL.InstRepos:28520533

\section{Terms of Use}

This article was downloaded from Harvard University's DASH repository, and is made available under the terms and conditions applicable to Other Posted Material, as set forth at http:// nrs.harvard.edu/urn-3:HUL.InstRepos:dash.current.terms-of-use\#LAA

\section{Share Your Story}

The Harvard community has made this article openly available.

Please share how this access benefits you. Submit a story.

\section{Accessibility}




\title{
An MRI study of temporal lobe abnormalities and negative symptoms in chronic schizophrenia
}

\author{
Jane E. Anderson ${ }^{\mathrm{a}}$, Cynthia G. Wiblea ${ }^{\mathrm{a}}$, Robert W. McCarleya, ${ }^{\mathrm{*}}$, Marianna Jakab ${ }^{\mathrm{b}}$, Kiyoto \\ Kasaia, and Martha E. Shenton $a, b,{ }^{*}$ \\ ${ }^{a}$ Clinical Neuroscience Division, Laboratory of Neuroscience, Department of Psychiatry, Brockton \\ Veterans Affairs Medical Center and Harvard Medical School, Boston, MA, USA \\ bSurgical Planning Laboratory, MRI Division, Brigham and Women's Hospital, Department of \\ Radiology, Harvard Medical School, Boston, MA, USA
}

\begin{abstract}
Previous magnetic resonance imaging (MRI) studies have reported various subtle brain abnormalities in schizophrenic patients, including temporal lobe abnormalities, which are of particular interest given the role of this brain region in auditory and language processing, and the characteristic deficits in these processes in schizophrenia. Subjects in this study were 16 male patients diagnosed with chronic schizophrenia and 15 healthy male comparison subjects. These patients were characterized by negative symptoms. High spatial resolution coronal MRI 1.5-mm-thick slices were used to measure the gray matter volume of the superior temporal gyrus, anterior and posterior amygdala/ hippocampal complex, and parahippocampal gyrus. Patients, relative to normal comparison subjects, evinced a reduction of gray matter volume in bilateral superior temporal gyri and anterior amygdala/ hippocampal complex. The reduction in gray matter of the superior temporal gyrus in patients with schizophrenia is consistent with previous findings, and is noteworthy in that it was found in this group of patients with predominantly negative symptoms. The reduction in the anterior amygdala/ hippocampal complex was an additional temporal lobe finding. These results underscore the role of temporal lobe structures in the pathophysiology of schizophrenia.
\end{abstract}

\section{Keywords}

Schizophrenia; Temporal lobe; Magnetic resonance imaging; Superior temporal gyrus; Amygdala; Hippocampus

\section{Introduction}

The temporal lobe has figured prominently in studies of schizophrenia, which is not surprising given the importance of this brain region for auditory and language processing — two processes that are notably abnormal in schizophrenia. Magnetic resonance imaging (MRI) studies have consistently reported temporal lobe abnormalities in schizophrenia (e.g., Johnstone et al., 1989; Bogerts et al., 1990; Becker et al., 1996; see also reviews by Shenton et al., 1997, 2001a,b; Pearlson, 1997; McCarley et al., 1999). Of particular note are MRI findings of Barta et al. (1990) of an association between auditory hallucinations with anteriorsuperior temporal

\footnotetext{
(C) 2002 Elsevier Science B.V. All rights reserved.

* Corresponding authors. M.E. Shenton and R.W. McCarley are to be contacted at: Department of Psychiatry-116A, Harvard Medical School, 940 Belmont Street, Brockton, MA 02301, USA. Robert_mccarley@hms.harvard.edu (R.W. McCarley),

Martha_Shenton@hms.harvard.edu (M.E. Shenton).
} 
gyrus (STG) and the findings of Shenton et al. (1992) of an association between posterior STG and thought disorder, which have spurred even greater interest in this brain region.

More specifically, there have been several studies reporting volume reductions in the gray matter of the STG (Shenton et al., 1992; Schlaepfer et al., 1994; Zipursky et al., 1994; Menon et al., 1995; Hajek et al., 1997; Hirayasu et al., 1998; Keshavan et al., 1998; Sullivan et al., 1998; Holinger et al., 1999; Marsh et al., 1999; Gur et al., 2000; Sanfilipo et al., 2000). Recent reviews (Shenton et al., 1997, 2001a,b; McCarley et al., 1999) document that all studies (listed above) evaluating STG gray matter, separate from white matter, have reported reductions in schizophrenic patients, and 10 out of 15 studies in which gray and white matter were combined have reported reductions in schizophrenic patients (positive findings: Barta et al., 1990, 1997; Flaum et al., 1995; Tune et al., 1996; Marsh et al., 1997; Pearlson et al., 1997; Reite et al., 1997; Keshavan et al., 1998; Bryant et al., 1999; Rajarethinam et al., 2000; negative findings: Vita et al., 1995; Kulynych et al., 1996; Woodruff et al., 1997; Roy et al., 1998; Havermans et al., 1999).

Structures of the medial temporal lobe have also been examined for their role in schizophrenia. The highly connected amygdala/hippocampal complex and parahippocampal gyrus are believed to be important for affective regulation and memory. Recent reviews (Shenton et al., 1997, 2001a,b; McCarley et al., 1999) describe reductions in medial temporal lobe structures as among the most consistent findings in the MRI literature. In particular, reductions of the amygdala/hippocampal complex have been widely reported (e.g., DeLisi et al., 1988; Barta et al., 1990; Bogerts et al., 1990; Breier et al., 1992; Shenton et al., 1992), but there have also been some negative findings in this region (e.g., Zipursky et al., 1994; Sanfilipo et al., 2000; Staal et al., 2000). In addition, reductions of the parahippocampal gyrus have been reported by some researchers (e.g., Shenton et al., 1992; Kawasaki et al., 1993) but not by others (e.g., Sanfilipo et al., 2000).

Hence, although there have been many findings of reductions in temporal lobe brain structures, there has been some lack of agreement among the studies. Such discrepancies may be due to differences in variables such as duration of illness, methodology, and symptom profile, the latter of which is the focus of the present study. We were interested in the distinction between positive symptoms (e.g., delusions, hallucinations, disorganized speech and behavior) and negative symptoms (e.g., attention deficits, flattened affect, social withdrawal, and poverty of speech or action) and whether temporal lobe structural abnormalities are specific to individuals whose symptoms are predominantly positive or whether they reflect a more general abnormality of schizophrenia (including those individuals with predominantly negative symptoms). We note, however, that there are other distinctions such as Liddle et al.'s (1989) three-factor division of symptoms into psychomotor poverty, disorganization, and reality distortion. As a first pass, however, we have focused on the more categorical classification of negative and positive symptoms, with an emphasis in this study on negative symptoms.

The goal of the current study was thus to evaluate temporal lobe brain abnormalities in a group of patients characterized primarily by negative symptoms. Hence, patients were recruited from outpatient community residences (where we tend to see more psychosocial morbidity due to negative symptoms) vs. the acute psychiatric wards of the hospital (where hospitalization has been required due to exacerbation of positive symptoms). We measured gray matter volumes of STG, amygdala/hippocampal complex, and parahippocampal gyrus in this chronic group of patients and in a comparison group of normal subjects. We also compared our findings with a previous group of patients with schizophrenia with more positive symptoms, and their respective control group, in order to evaluate possible differences in temporal lobe abnormalities between patients with more negative versus more positive symptoms. Below, we report our findings in schizophrenia where we document temporal lobe volume reductions 
in the bilateral superior temporal gyri and anterior amygdala/hippocampal complex in this group of patients with predominantly negative symptoms.

\section{Methods}

\subsection{Subjects}

Sixteen right-handed male patients diagnosed with schizophrenia and from the VA Boston Healthcare System, Brockton Division, in Massachusetts participated in the study. All patients were diagnosed with schizophrenia based on the Diagnostic and Statistical Manual of Mental Disorders III-R (APA, 1987) criteria, derived from the Structured Clinical Interview for DSMIII-R (Spitzer et al., 1990), and from information obtained from psychiatric records. All subjects were receiving neuroleptic medication equivalent to a mean of $347 \mathrm{mg}$ of chlorpromazine per day, one was hospitalized at the time of recruitment, and 15 were in VA community care homes. The mean age of patients was $42.50 \pm 8.79$ and their mean duration of illness was 19.69 years \pm 8.57 .

The age range for inclusion in the study was 20 to 55 years. Subjects were excluded if they had a history of: (1) neurologic illness or major head trauma; (2) electroconvulsive therapy; (3) alcohol or drug dependence; or (4) alcohol and drug abuse within the past 5 years. The normal comparison group of 15 right-handed males was recruited through a newspaper advertisement and screened to exclude neurologic and psychiatric illness and alcohol abuse in themselves or in their first degree relatives. The mean age of the normal comparison group was $41.67 \pm 7.51 \mathrm{SD}$. None of the patients or comparison subjects had participated in our earlier study of the same ROIs (Shenton et al., 1992). All subjects gave written informed consent prior to study participation.

Handedness was assessed using the Edinburgh inventory (Oldfield, 1971; schizophrenia subjects: $0.79 \pm 0.16$; normal comparison subjects: $0.83 \pm 0.16)$ and parental socioeconomic status was measured by the Hollingshead two-factor index (Hollingshead, 1965: 1 = highest, 5 = lowest; schizophrenia subjects: $3.31 \pm 1.14$; normal comparison subjects: $2.67 \pm 0.82$ ). All subjects were given the WAIS-R information subscale (Wechsler, 1981; schizophrenia subjects: $10.63 \pm 2.33$; normal comparison subjects: $11.33 \pm 1.88$ ) as an estimate of gross fund of information.

\subsection{Clinical measures}

The following tests were administered to patients: The Scale for the Assessment of Positive Symptoms (SAPS; Andreasen, 1984), The Scale for the Assessment of Negative Symptoms (SANS; Andreasen, 1981), the Positive and Negative Syndrome Scale (PANSS; Kay et al., 1987), and the Thought Disorder Index (e.g., Johnston and Holzman, 1979; Solovay et al., 1987).

\subsection{MRI acquisition and processing}

MRI scans were obtained using a 1.5-T General Electric Signa System (GE Medical Systems, Milwaukee) at the Brigham and Women's Hospital in Boston, MA. For measuring the volume of the total intracranial contents [brain, cerebral spinal fluid (CSF), connective tissue and blood vessels], a continuous series of double-echo spin-echo axial slices (proton density and T2 weighted) was obtained (after a sagittal localizer), with the following imaging parameters: repetition time $=3000 \mathrm{~ms}$, echo time $=30$ and $80 \mathrm{~ms}$, field of view $=24 \mathrm{~cm}$, matrix $=256 \times$ 256 (192 phase encoding steps, with zero filling), and an interleaved acquisition with $3-\mathrm{mm}$ slice thickness. Voxel dimensions were $0.9375 \times 0.9375 \times 3 \mathrm{~mm}$. 
For the manual measurement of temporal lobe structures, three-dimensional Fourier-transform (3DFT) spoiled gradient-recalled acquisition (SPGR) were obtained with the following parameters: repetition time $=35 \mathrm{~ms}$, echo time $=5 \mathrm{~ms}$, one repetition, $45^{\circ}$ nutation angle, 24 $\mathrm{cm}$ field of view, matrix $=256 \times 256(192$ phase encoding steps $) \times 124$, and an interleaved acquisition with $1.5-\mathrm{mm}$ slice thickness. Voxel dimensions were $0.9375 \times 0.9375 \times 1.5 \mathrm{~mm}$. The 124 slices, $1.5-\mathrm{mm}$ thick, were formatted in the coronal plane.

For both axial (double echo spin-echo) and coronal (SPGR) acquisitions, gradient moment nulling and presaturation of a slab inferior to the head were performed to reduce flow-related artifacts. To reduce noise, an anisotropic diffusion filter was used prior to processing each set of scans (Gerig et al., 1992). In addition, the anterior commissure/posterior commissure (AC/ PC) line was used to align the scans prior to acquisition.

To measure total intracranial contents, a semiautomated segmentation algorithm (Cline et al., $1988,1990)$ was applied to the axial double echo images. This algorithm assigns voxels to distinct tissue classes (i.e., gray matter, white, matter, CSF, and connective tissue and vessels) based on signal intensities. A detailed description of this procedure can be found in Shenton et al. (1992).

\subsection{Definition of regions of interest}

The superior temporal gyrus, amygdala/hippocampal complex and parahippocampal gyrus were outlined manually on a Sun workstation. The most anterior slice used for the superior temporal gyrus and amygdala/hippocampal complex was the one in which the white matter tract linking the temporal lobe with the rest of the brain (temporal stem) could be seen. The most posterior slice was the last appearance of fibers of the crux of the fornix. The posterior segment of the STG and the amygdala/hippocampal complex began with the first appearance of the mammillary bodies. The parahippocampal gyrus (non-subicular portions) was defined laterally by the collateral sulcus and a demarcation line drawn across the narrow portion of the gyral isthmus at the deepest portion of the collateral sulcus (for more detailed information see Shenton et al., 1992).

All editing was done blind to diagnosis (but not to hemisphere as our raters are sufficiently sophisticated that they would discern major neuroanatomical differences between the hemispheres such as differences in the Sylvian fissure). Interrater reliability of the ROIs was evaluated by three independent raters (JEA, TK, SC) who were also blind to diagnosis (a fourth rater $[\mathrm{KK}]$ was included for the STG). Ten cases were selected at random and every third slice was edited by the raters. Intraclass correlation coefficients were computed for each of manual drawings of the regions of interest and yielded the following intraclass coefficients: STG: $r=$ 0.82; amygdala/hippocampal complex: $r=0.95$; parahippocampal gyrus: $r=0.77$.

\subsection{Statistical analyses}

Independent sample $t$-tests were used to test for differences in age, handedness score, parental socioeconomic status, and WAIS-R information subscale scores. Total ICC was used to compute relative volumes in order to control for variations in brain size [100 $\times($ ROI volume/ ICC volume)]. In addition to using this ratio to control for differences in brain size, we also performed linear regression measures on the absolute volume to adjust for total ICC. Because both methods are used in the literature, we included both for comparison here. Statistical results of the analyses of residual volumes are mentioned only if they differed from those of the relative volume. Statistical analyses were based on the relative and residual volumes. Percent differences were calculated from relative volume measures. 
To determine whether or not certain ROIs were more reduced than other ROIs, the ROI volumes were first converted to $Z$-scores so that all the ROIs would be on the same scale. This was necessary because ROIs differ from one another in size, hence a larger structure (e.g., the STG) is likely to show a larger difference than a smaller structure (e.g., the parahippocampal gyrus) simply because it is a larger structure to begin with, but not necessarily because it is more greatly affected. The mean and standard deviation of the comparison group were used to calculate the $Z$-scores and the normalized scores were then submitted to an ANOVA with two within subject variables, ROI (STG, anterior amygdala/hippocampal complex, posterior amygdala/hippocampal complex, parahippocampal gyrus) and side (left, right), and one between subject variable, group (schizophrenia, comparison). Follow-up ANOVAs were conducted on the absolute and relative volumes of each ROI with side (left, right) as a within subject variable and group as a between subjects variable. The Huynh-Feldt correction was applied to all analyses with greater than 1 degree of freedom in the numerator. Because our a priori hypothesis was that the ROIs would differ between schizophrenia and comparison groups, as they did in the previous study (Shenton et al., 1992), planned comparisons using $t$ tests were used to compare volumes, however, we conservatively chose to use two-tailed tests of significance.

Pearson correlations were used to examine the relationship between the temporal lobe ROIs. Two-tailed tests of significance were utilized on both absolute and relative volumes, with a conservative significance level of 0.001 (Bonferroni correction). Because the distribution of clinical measures was not normal, exploratory analyses of the correlations between ROIs and clinical measures were evaluated using Spearman rank-order correlation coefficients (twotailed tests with a significance level conservatively set at 0.01 ).

To compare the symptom profile of the patients in the present study with that of the patients in the previous study from our lab (Shenton et al., 1992), a repeated measures ANOVA was done with test score (total score from the SAPS, total score from SANS) as a within subjects variable and group (present study, previous study) as a between subjects variable.

In order to examine further the role of symptomatology, we combined the previous data set (Shenton et al., 1992) with the current data set. Z-scores of ROI volumes were used for several reasons. First, the patients were from separate studies with different comparison groups. Secondly, although the acquisition parameters were identical, there had been several upgrades of the software to the GE scanner since the 1992 study. Finally, while we used the same neuroanatomical landmarks for the ROI measures, different raters were used in the earlier and later studies, again suggesting that it would be appropriate to use $Z$-scores. The $Z$-scores were computed for each patient by using the mean and standard deviation of the corresponding comparison group for that patient. Using the $Z$-score adjusted volumes, correlation analyses were completed for total SAPS and SANS scores and ROI volumes. In addition, a median split was performed to separate the combined pool of subjects into two groups - those with low SAPS scores and those with high SAPS scores, and $t$-tests were conducted to compare the $Z$ score-adjusted ROI volumes for these two groups. (The same analyses were conducted using a median split of total SANS scores.) We also did a median split of the pooled data based on TDI totals and compared ROI volumes using $t$-tests.

\section{Results}

There were no significant group differences in age, handedness score (Edinburgh inventory, Oldfield, 1971), parental socioeconomic status (Hollingshead two-factor index; Hollingshead, 1965), or information subscale of the WAIS (Wechsler, 1981). 


\subsection{Temporal lobe regions of interest}

There were no significant differences between groups in volume of the total intracranial contents $[t(29)=-1.024, p=0.314]$. The repeated measures ANOVA of normalized ROI values (Z-scores) with ROI (STG, anterior amygdala/hippocampal complex, posterior amygdala/hippocampal complex, and parahippocampal gyrus) and side (left, right) as within subjects variables and group as a between subjects variable revealed a trend for an interaction of ROI and group $[F(3,87)=2.348, p<0.087)$. To further examine this trend, each ROI was compared between groups separately. Significant differences were found for the STG $[F(1,29)$ $=5.384, p<0.028 ;$ an $8 \%$ difference $]$ and anterior amygdala/hippocampal complex $[F(1,29)$ $=4.857, p<0.036 ;$ a $13 \%$ difference], but not for the posterior amygdala/hippocampal complex or parahippocampal gyrus. There were no Group by Hemisphere interactions, indicating a lack of lateralization of the effects.

Planned comparisons of individual ROIs revealed that the left superior temporal gyrus volume was reduced in the patient group by $9 \%[t(29)=-2.626, p<0.014$; see Fig. 1]. The anterior portion of the left STG was smaller (by 25\%) in the patient group, a difference that approached significance $[t(29)=-1.790, p=0.073]$. A $6 \%$ reduction in the left posterior portion did not reach significance $(p=0.11)$.

There were no significant differences among the individual $t$-tests of the other medial temporal lobe structures (left and right anterior amygdala/hippocampus, posterior amygdala/ hippocampus, and parahippocampal gyrus). Table 1 lists the means of each ROI and corresponding $p$-values from the $t$-tests. There were no significant correlations between chlorpromazine equivalent and any of the temporal lobe regions of interest.

It is likely that the differences found in the anterior left and posterior STG may have been only marginal due to a lack of statistical power. In fact, a post hoc power analysis shows that if the number of subjects were increased to approximately 22 subjects per group, the results would likely have reached significance at the 0.05 level. Furthermore, given the directional nature of our hypothesis, one could argue that one-tailed $t$-tests were warranted here. If one-tailed tests were used, the left anterior STG would have reached significance $(p<0.037$, while the left posterior STG and right anterior amygdala/hippocampal complex would have still only approached significance ( $p=0.056$ and $p=0.053$, respectively).

In addition to the analyses of absolute and relative volume measures, we also performed analyses using residual measures from a regression analysis controlling for total ICC. The results of the ANOVA and $t$-tests were consistent with those of the relative volumes.

\subsection{Correlations among regions of interest}

Correlations are reported for absolute volumes, but were considered significant only if they reached a significance level of 0.001 for both absolute and relative volumes. The schizophrenia group showed a positive correlation between the left and right parahippocampal gyrus ( $r=$ $0.80, p<0.0005$ ), while the normal comparison group also showed a positive correlation of the relative volume of the left and right parahippocampal gyrus $(r=0.82, p<0.0005)$ but only a trend for the absolute volume $(r=0.74, p<0.002)$. There were no other significant correlations found in the subjects with schizophrenia. [In the normal comparison group there were several positive correlations: left and right posterior STG $(r=0.82, p<0.0005)$; left anterior amygdala/hippocampal complex and left anterior STG $(r=0.80, p<0.0005)$; and right anterior amygdala/hippocampal complex and right anterior STG $(r=0.80, p<0.0005)]$. 


\subsection{Correlations with clinical measures}

The correlation between right posterior STG and "poor attention" subscale of the PANSS (rho $=-0.67, p<0.005$ ) was the only subscale from the PANSS, SAPS or SANS to reach our criterion for significance ( $p<0.01$ for both absolute and relative volumes).

Scores on the TDI were available for eleven subjects with schizophrenia. We found a negative correlation between the degree of thought disorder and absolute volume of left STG (rho = $0.606, p<0.048$ ). There was also a negative correlation between the absolute volume of right posterior STG and TDI (rho $=-0.610, p=0.046$ ). Positive correlations were found between the TDI and left parahippocampal gyrus and for both absolute (rho $=0.615, p=0.044$ and relative volumes (rho $=0.624, p=0.040$ ). The correlation of TDI and absolute volume of the right parahippocampal gyrus approached significance $($ rho $=0.587, p<0.058)$, while the relative volume reached significance ( rho $=0.692, p<0.018$ ).

\subsection{Symptom profile comparison}

As noted previously, we recruited patients whose symptom profile was characterized by more negative symptoms vs. positive symptoms. To quantify differences in the symptom profile of the previous subject sample (Shenton et al., 1992) and the present sample, an ANOVA was done with repeated measures ANOVA with score (SAPS, SANS) as a within subjects variable and group (previous, present patients with schizophrenia) as a between subjects variable. There was a significant interaction of score and group $(F(1,28)=4.761, p<0.038)$. [There was no main effect of score $(p=0.146)]$. The interaction was a cross-over interaction (illustrated in Fig. 2) in which the total SAPS was greater in the Shenton et al. (1992) sample while the total SANS was greater in the present sample. This finding highlights the difference between the two samples in symptom profiles and confirms that our attempts to recruit patients, characterized by more negative symptoms compared to our previous sample, were indeed successful.

\subsection{Combined data from previous study}

Subjects from the present study were pooled with those from our previous study (Shenton et al., 1992) in order to examine the relationship between symptom profile and brain morphology. ROI volumes were converted to $Z$-scores (see Methods). There was a trend towards a significant negative correlation between the left posterior STG and total SAPS score (Z-score adjusted volume: $r=-0.33, p<0.078$ ), such that the higher the total SAPS score, the lower the left posterior STG volume. To further investigate this relationship, we did a median split of total SAPS scores on the pooled subjects from both the present study and the previous study (Shenton et al., 1992) and compared Z-score adjusted volumes of the left posterior STG. Although the analysis did not reach significance $(p=0.152)$, the effect size was large $(0.55)$ and the direction of the finding was consistent with the above negative correlation in that subjects with higher total SAPS scores had lower volume of left posterior STG. SANS total scores did not correlate significantly with any of the $Z$-score-adjusted ROIs, nor were there any significant findings in the $t$-tests of $Z$-score-adjusted ROIs based on the median split of SANS total scores. The median split of the TDI scores revealed that subjects with a greater degree of thought disorder had lower volumes in left STG $[t(20)=3.555, p<0.002]$, both its anterior $[t(20)=2.805, p<0.011$ and posterior regions $[t(20)=2.581, p<0.018]$, and left anterior amygdala/hippocampal complex $[t(20)=2.838, p<0.01]$ compared to those with a lesser degree of thought disorder.

\section{Discussion}

The major findings of this study were MRI gray matter volume reduction in bilateral STG and anterior amygdala/hippocampal complex in a group of patients with chronic schizophrenia manifesting mainly negative symptoms. The STG finding is consistent with several other 
studies (e.g., Zipursky et al., 1994; Menon et al., 1995; Marsh et al., 1999; Gur et al., 2000; Sanfilipo et al., 2000), which have found bilateral STG reduction in individuals with schizophrenia. The superior temporal gyrus is a brain region that is important for auditory processing and also plays a significant role in language processing (which is usually leftlateralized in right-handed individuals). Hence, it is not surprising that this region would be affected in schizophrenia.

In the previous study from our lab (Shenton et al., 1992), volume reductions were found in a schizophrenia group evincing predominantly positive symptoms in the posterior region of the left STG, while in the present study the volume reduction was not localized to the left posterior portion but was significant in the STG as a whole. However, it should be noted that in the present study the effect size was large in the left STG (0.91) but only moderate in the right STG (0.53). In addition, the $t$-tests of individual ROIs revealed a significant reduction in the left STG but not in the right STG. This pronounced effect in the left STG is thus consistent with our previous study as well as other studies that have found left STG reductions in schizophrenia (e.g., Hirayasu et al., 1998;Keshavan et al., 1998).

The absence of significant correlations between STG volume and neuroleptic dosage suggests that STG volume changes were not secondary to neuroleptic dosage. Furthermore, Hirayasu et al. (1998) have reported left STG volume reductions in patients newly diagnosed with schizophrenia who had received neuroleptic treatment for a brief time only (less than 2 months). In addition, the STG volume reduction found in the Hirayasu et al. study was specific to patients with schizophrenia and it was not observed in a group of bipolar patients.

The STG finding is hence a robust finding appearing in both chronic and first episode patients. Furthermore, unlike many other MRI findings, it appears consistently across studies from multiple laboratories using a variety of methodologies and patient populations. An important finding from the present study is that STG reduction does not appear to be dependent upon the symptom profile (i.e., positive or negative symptom predominance). It is important to point out, however, that the positive/negative distinction is thought to be dimensional rather than categorical (Andreasen and Olson, 1982), and that although patients in this study had a predominance of negative symptoms, they still manifested positive symptoms.

The reduction in anterior amygdala/hippocampal complex is consistent with other studies (e.g., Breier et al., 1992; Marsh et al., 1994). This anterior region of the amygdala/hippocampal complex largely encompasses the amygdala, a structure thought to be important for heightening the processing of information during emotional states (Bechara et al., 1995; Gloor, 1986; see also Wible et al., 1997). Findings in this region have been mixed regarding lateralization of the effect. Pearlson et al. (1997) found reduced volume in the right amygdala in schizophrenic subjects, while Shenton et al. (1992) found volume reduction in the left anterior amygdala/ hippocampal complex. In our present study, the effect was not lateralized to either hemisphere. Some studies have not found any differences in this region (e.g., Swayze et al., 1992; Becker et al., 1996; Staal et al., 2000). Mixed findings may be due to methodological differences such as different segmentation and measurement techniques, and different slice thicknesses, as well as differing symptom profiles of patients.

Reductions in other medial temporal lobe structures have also been widely reported in the literature (for reviews see Shenton et al., 1997, 2001a,b; McCarley et al., 1999). For example, volume reductions in the hippocampus have been reported by Bogerts et al. $(1990,1993)$, especially among male patients and in the left hemisphere (see also Becker et al., 1996). In addition, the volume of the parahippocampal gyrus has been found to be reduced in schizophrenic patients (Shenton et al., 1992). However, not all studies have found significant differences in the hippocampus region (e.g., Zipursky et al., 1994; Sanfilipo et al., 2000) or 
parahippocampal gyrus (e.g., Sanfilipo et al., 2000). In the present study, we did not find significant differences in the posterior hippocampus or in parahippocampal gyrus. The interrater reliability was low for the parahippocampal gyrus, a factor which may have contributed to the null finding.

In sum, reductions in medial temporal lobe structures have been widely reported but there are inconsistencies in the literature regarding the particular structures, however, reductions in STG gray matter have an extremely high degree of replicability. As noted in the introduction, all of the studies that have measured gray matter of STG have found reductions in schizophrenia compared to normal comparison subjects (Shenton et al., 1997, 2001a,b; McCarley et al., 1999).

There were several differences between the present findings and those of the previous study from our lab (Shenton et al., 1992). Our finding of STG reduction approximated that of the previous finding of left posterior STG reduction in that our effect size was more pronounced in the left hemisphere. In addition, our anterior amygdala/hippocampal complex finding was bilateral compared to the previous finding of reductions in left anterior amygdala/hippocampal complex. Furthermore, while the previous study found reductions in the left and right parahippocampal gyrus, we did not find any differences in these structures. The image processing tools were the same in both studies with the exception of upgrades made to the scanner. One possible reason for the slightly different findings is that our new sample of patients differed in clinical symptomatology from our previous sample. The current sample was characterized by less severe positive symptoms, and more severe negative symptoms. An ANOVA of SAPS and SANS total score revealed a cross-over interaction, which is illusrated in Fig. 2. Although positive and negative symptoms may fluctuate in terms of acute exacerbations of the illness, the patients (in both samples) were chronic patients who tend to have a more stable symptom profile over time. When we combined the current data with the previous chronic patient sample, we found a trend for positive symptoms being associated with left STG reductions.

Shenton et al. (1992) reported several correlations among temporal lobe structures (e.g., between the left parahippocampal gyrus and the left posterior amygdala/hippocampal complex, and the left anterior STG and the left anterior amygdala/hippocampal complex) that were present in the schizophrenia group but not in the comparison group, however, these correlations did not reach significance in the present study. It is unclear why we did not find similar correlations among our subjects with schizophrenia, but one likely possibility is the different symptom profiles between the patients in the two studies.

In the present study we examined the relationship between clinical measures and temporal lobe structures. While several previous studies have reported negative correlations between the severity of hallucinations and left STG (Barta et al., 1990; Flaum et al., 1995; Levitan et al., 1999; Rajarethinam et al., 2000), as in our previous study (Shenton et al., 1992), we did not find such a correlation. Furthermore, while some studies (e.g., Shenton et al., 1992; Menon et al., 1995; Marsh et al., 1997) have found reported a negative correlation between severity of thought disorder and left posterior STG volume reduction, we observed a negative correlation with severity of thought disorder and both left STG and right posterior STG volume reduction (absolute volume only). When the two patient groups (from the current study and Shenton et al., 1992) were pooled together and split into two groups based on TDI score, those with a higher degree of thought disorder had lower volumes in left STG compared to those with lesser degrees of thought disorder. In addition, we found a negative correlation between the right posterior STG and the "poor attention" subscale of the PANSS, a finding that may be linked to the fact that our patient sample displayed predominant negative symptoms. It is important 
to point out that our correlation analyses were exploratory in nature, hence should be interpreted with caution until replicated and subjected to more conservative tests.

One limitation of the study is that it did not include any female subjects because patients were recruited from a Veterans Affairs Medical Center. In future studies, it will be important to compare the volumes of temporal lobe structures of both female and male subjects with corresponding normal comparison groups. Furthermore, patients with a greater range of symptoms (both positive and negative) should be included to further study the role of symptomatology.

It will also be important in future studies to more closely examine the nature of language disturbances in schizophrenia and to determine if they are associated with volume reduction in specific regions of the STG. Recent evidence from neuroimaging tools (e.g., PET and fMRI) has led to exciting new hypotheses of language processing in the brain that link specific neuroanatomic areas of the STG with specific language functions. For example, Hickok and Poeppel (2000) (see also Buchsbaum et al., in press) propose that there are functionally distinct regions in the posterior STG involved in speech processing that may comprise divergent dorsal and ventral processing systems. According to this view, the dorsal stream projects from the posterior STG/parietal operculum to Broca's area and interfaces sound-based representations of speech with motor-based representations. The ventral stream projects from posterior superior temporal sulcus posteriorly to temporal-parietal-occipital structures and interfaces sound-based representations with conceptual representations. Another view, espoused by Wise et al. (2001), postulates two subsystems that are important for the acquisition of new lexical representations: One, in the left posterior superior temporal sulcus, is important for processing the temporal order of sounds that make up heard or internally generated words. The other, in the nearby left medial temporoparietal junction, is involved in the repetition of words and sounds. New models such as these invite more in-depth studies in the fields of functional and structural neuroimaging that take a closer look at the functional significance of subregions of the STG.

In conclusion, we found further evidence of abnormal temporal lobe structures (STG and anterior amygdala/hippocampal complex) in patients with chronic schizophrenia with predominantly negative symptoms. The findings are consistent with previous MRI studies and further establish the temporal lobe as a significant region in the study of schizophrenia.

\section{Acknowledgments}

The authors gratefully acknowledge the assistance of Sare Akdag and Joanna Gainski for their recruitment of participants, Tanya Kisler and Sarah Corriveau for their technical assistance, Ferenc Jolesz, M.D. for his helpful comments, and Christopher Allen and Marie Fairbanks for their administrative assistance.

This research was supported in part by the National Institute of Mental Health through an Individual National Service Research Award, MH 11939 (JEA), by the National Institute of Mental Health MH R01 50740 (MES), MH K02 01110 (MES), NIMH 40977 (RWM); by two individual Veterans Administration Merit Awards (MES; RWM), and by a Basic and Clinical Neuroscience Research Center Grant, and the Department of Veterans Affairs (RWM).

\section{References}

American Psychiatric Association. Diagnostic and Statistical Manual of Mental Disorders. 3rd edn.. Rev: DSM-III-R American Psychiatric Press; Washington, DC: 1987.

Andreasen, NC. Scale for the Assessment of Negative Symptoms (SANS). Department of Psychiatry, University of Iowa College of Medicine; Iowa City, IA: 1981.

Andreasen, NC. Scale for the Assessment of Positive Symptoms (SAPS). Department of Psychiatry, University of Iowa College of Medicine; Iowa City, IA: 1984. 
Andreasen NC, Olson S. Negative and positive schizophrenia: definition and validation. Arch. Gen. Psychiatry 1982;39:789-793. [PubMed: 7165478]

Barta PE, Pearlson GD, Powers RE, Richards SS, Tune LE. Auditory hallucinations and smaller superior temporal gyral volume in schizophrenia. Am. J. Psychiatry 1990;147:1457-1462. [PubMed: 2221156]

Barta PE, Powers RE, Aylward EH, Chase GA, Harris GJ, Rabins PV, et al. Quantitative MRI volume changes in late onset schizophrenia and Alzheimer's disease compared to normal controls. Psychiatry Res 1997;68:65-75. [PubMed: 9104754]

Bechara A, Tranel D, Damasio H, Adolphs R, Rockland C, Damasio AR. Double dissociation of conditioning and declarative knowledge relative to the amygdala and hippocampus in humans. Science 1995;269:1115-1118. [PubMed: 7652558]

Becker T, Elmer K, Schneider F, Schneider M, Grodd W, Bartels M, et al. Confirmation of reduced temporal limbic structure volume on magnetic resonance imaging in male patients with schizophrenia. Psychiatry Res 1996;67:135-143. [PubMed: 8876013]

Bogerts B, Ashtari M, Degreef G, Alvir JM, Bilder RM, Lieberman JA. Reduced temporal limbic structure volumes on magnetic resonance images in first episode schizophrenia. Psychiatr. Res.: Neuroimaging 1990;35:1-13.

Bogerts B, Lieberman JA, Ashtari M, Bilder RM, Degreef G, Lerner G, et al. Hippocampus-amygdala volumes and psychopathology in chronic schizophrenia. Biol. Psychiatry 1993;33:236-246. [PubMed: 8471676]

Breier A, Buchanan RW, Elkashef A, Munson RC, Kirkpatrick B, Gellad F. Brain morphology and schizophrenia: a magnetic resonance imaging study of limbic, prefrontal cortex, and caudate structures. Arch. Gen. Psychiatry 1992;49:921-926. [PubMed: 1449382]

Bryant NL, Buchanan RW, Vladar K, Breier A, Rothman M. Gender differences in temporal lobe structures of patients with schizophrenia: a volumetric MRI study. Am. J. Psychiatry 1999;156:603609. [PubMed: 10200741]

Buchsbaum B, Hickok G, Humphries C. Role of left posterior superior temporal gyrus in phonological processing for speech perception and production. In press.

Cline HE, Lorensen WE, Ludke S, Crawford CR, Teeter BC. Two algorithms for the three-dimensional reconstruction of tomograms. Med. Phys 1988;15:320-327. [PubMed: 3043154]

Cline HE, Lorensen WE, Kikinis R, Jolesz FA. Three-dimensional segmentation of MR images of the head using probability and connectivity. J. Comput. Assist. Tomogr 1990;14:1037-1045. [PubMed: 2229557]

DeLisi LE, Dauphinais D, Gershon ES. Perinatal complications and reduced size of brain limbic structures in familial schizophrenia. Schizophr. Bull 1988;14:185-191. [PubMed: 3201176]

Flaum M, Swayze VW II, O'Leary DS, Yuh WTC, Ehrhardt JC, Arndt SV, et al. Effects of diagnosis and gender on brain morphology in schizophrenia. Am. J. Psychiatry 1995;152:704-714. [PubMed: 7726310]

Gerig G, Kubler O, Kikinis R, Jolesz F. Non-linear anisotropic filtering of MRI data. IEEE Trans. Med. Imaging 1992;11:221-232. [PubMed: 18218376]

Gloor, P. The role of the human limbic system in perception, memory and affect. In: Doane, BK.; Livingston, KE., editors. The Limbic System. Raven Press; New York: 1986. p. 95-108.

Gur RE, Turetsky BI, Cowell PE, Finkelman C, Maany V, Grossman RI, et al. Temporolimbic volume reductions in schizophrenia. Arch. Gen. Psychiatry 2000;57:769-775. [PubMed: 10920465]

Hajek M, Huonker R, Boehle C, Volz HP, Nowak H, Sauer H. Abnormalities of auditory evoked magnetic fields and structural changes in the left hemisphere of male schizophrenic: a magnetoencephalographic-magnetic resonance imaging study. Biol. Psychiatry 1997;42:609-616. [PubMed: 9376457]

Havermans R, Honig A, Vuurman EF, Krabbendam L, Wilmink J, Lamers T, et al. A controlled study of temporal lobe structure volumes and P300 responses in schizophrenic patients with persistent auditory hallucinations. Schizophr. Res 1999;38:151-158. [PubMed: 10463460]

Hickok G, Poeppel D. Towards a functional neuroanatomy of speech perception. Trends Cognit. Sci 2000;4:131-138. [PubMed: 10740277]

Hirayasu Y, Shenton ME, Salisbury DF, Dickey CD, Fischer IA, Kisler T, et al. Lower left temporal lobe MRI volmes patients with first-episode schizophrenia compared with psychotic patients with first- 
episode affective disorder and normal subjects. Am. J. Psychiatry 1998;155:1384-1391. [PubMed: 9766770]

Holinger DP, Shenton ME, Wible CG, Donnino R, Kikinis R, Jolesz F, et al. Superior temporal gyrus volume abnormalities and thought disorder in left-handed schizophrenic men. Am. J. Psychiatry 1999;156:1730-1735. [PubMed: 10553736]

Hollingshead, AB. Two-Factor Index of Social Position. Yale University Press; New Haven, CN: 1965.

Johnston, MH.; Holzman, PS. Assessing Schizophrenic Thinking. Jossey-Bass; San Francisco, CA: 1979.

Johnstone EC, Owens DGC, Crow TJ, Frith CD, Alexandropolis K, Bydder G, et al. Temporal lobe structure as determined by nuclear magnetic resonance in schizophrenia and bipolar affective disorder. J. Neurol., Neurosurg. Psychiatry 1989;52:736-741. [PubMed: 2746266]

Kawasaki Y, Maeda Y, Urata K, Higashima M, Yamaguchi N, Suzuki M, et al. A quantitative magnetic resonance imaging study of patients with schizophrenia. Eur. Arch. Psychiatry Clin. Neurosci 1993;242:268-272. [PubMed: 8499495]

Kay SR, Fiszbein A, Opler LA. The positive and negative syndrome scale (PANSS) for schizophrenia. Schizophr. Bull 1987;13:261-276. [PubMed: 3616518]

Keshavan MS, Haas GL, Kahn CE, Aguilar E, Dick EL, Schooler NR, et al. Superior temporal gyrus and the course of early schizophrenia: progressive, static, or reversible? J. Psychiatr. Res 1998;32:161167. [PubMed: 9793869]

Kulynych JJ, Vladar K, Jones DW, Weinberger DR. Superior temporal gyrus volume in schizophrenia: a study using MRI morphometry assisted by surface rendering. Am. J. Psychiatry 1996;153:50-56. [PubMed: 8540591]

Levitan C, Ward PB, Catts SV. Superior temporal gyral volumes and laterality correlates of auditory hallucinations in schizophrenia. Biol. Psychiatry 1999;46:955-962. [PubMed: 10509178]

Liddle PF, Barnes TP, Morris D, Haque S. Three syndromes in chronic schizophrenia. Br. J. Psychiatry 1989;(Suppl 7):119-122.

Marsh L, Suddath RL, Higgins N, Weinberger DR. Medial temporal lobe structures in schizophrenia: relationship of size to duration of illness. Schizophr. Res 1994;11:225-238. [PubMed: 8193061]

Marsh L, Harris D, Lim KO, Beal M, Hoff AL, Minn K, et al. Structural magnetic resonance imaging abnormalities in men with severe chronic schizophrenia and an early age at clinical onset. Arch. Gen. Psychiatry 1997;54:1104-1112. [PubMed: 9400346]

Marsh L, Lim KO, Hoff AL, Harris D, Beal M, Minn K, et al. Severity of schizophrenia and magnetic resonance imaging abnormalities: a comparison of State and Veterans hospital patients. Biol. Psychiatry 1999;45:49-61. [PubMed: 9894575]

McCarley RW, Wible CG, Frumin M, Hirayasu Y, Levitt JJ, Fischer IA, Shenton ME. MRI anatomy of schizophrenia. Biol. Psychiatry 1999;45:1099-1119. [PubMed: 10331102]

Menon RR, Barta PE, Aylward EH, Richards SS, Vaughn DD, Tien AY, et al. Posterior superior temporal gyrus in schizophrenia: grey matter changes and clinical correlates. Schizophr. Res 1995;16:127135. [PubMed: 7577766]

Oldfield RC. The assessment and analysis of handedness: the Edinburgh inventory. Neuropsychologia 1971;9:97-113. [PubMed: 5146491]

Pearlson GD. Superior temporal gyrus and planum temporale in schizophrenia: a selective review. Prog. Neuropsychopharmacol. Biol. Psychiatry 1997;21:1203-1229. [PubMed: 9460087]

Pearlson GD, Barta PE, Powers RE, Menon RR, Richards SS, Aylward EH, et al. Medial and superior temporal gyral volumes and cerebral asymmetry in schizophrenia versus bipolar disorder. Biol. Psychiatry 1997;41:1-14. [PubMed: 8988790]

Rajarethinam RP, DeQuardo JR, Nalepa R, Tandon R. Superior temporal gyrus in schizophrenia: a volumetric magnetic resonance imaging study. Schizophr. Res 2000;41:303-312. [PubMed: 10708339]

Reite M, Sheeder J, Teale P, Adams M, Richardson D, Simon J, et al. Magnetic source imaging evidence of sex differences in cerebral lateralization in schizophrenia. Arch. Gen. Psychiatry 1997;54:433440. [PubMed: 9152097]

Roy PD, Zipursky RB, Saint-Cyr JA, Bury A, Langevin R, Seeman MV. Temporal horn enlargement is present in schizophrenia and bipolar disorder. Biol. Psychiatry 1998;44:418-422. [PubMed: 9777171] 
Sanfilipo M, Lafargue T, Rusinek H, Arena L, Loneragan C, Lautin A, et al. Volumetric measure of the frontal and temporal lobe regions in schizophrenia: relationship to negative symptoms. Arch. Gen. Psychiatry 2000;57:471-480. [PubMed: 10807487]

Schlaepfer TE, Harris GJ, Tien AY, Peng LW, Lee S, Federman EB, et al. Decreased regional cortical gray matter volume in schizophrenia. Am. J. Psychiatry 1994;151:842-848. [PubMed: 8184992]

Shenton ME, Kikinis R, Jolesz FA, Pollak SD, LeMay M, Wible CG, et al. Abnormalities of the left temporal lobe and thought disorder in schizophrenia. N. Engl. J. Med 1992;327:604-612. [PubMed: 1640954]

Shenton, ME.; Wible, CG.; McCarley, RW. A review of magnetic resonance imaging studies of brain abnormalities in schizophrenia. In: Krishnan, KRR.; Doraiswamy, PM., editors. Brain Imaging in Clinical Psychiatry. Marcel Dekker; New York: 1997. p. 297-380.

Shenton ME, Dickey CC, Frumin M, McCarley RW. A review of MRI findings in schizophrenia. Schizophr. Res 2001a;49:1-52. [PubMed: 11343862]

Shenton, ME.; Frumin, M.; McCarley, RW.; Maier, S.; Westin, CF.; Fischer, IA., et al. Morphometric magnetic resonance imaging studies: findings in schizophrenia. In: Dougherty, DD.; Rauch, SL., editors. Psychiatric Neuroimaging Research: Contemporary Strategies. American Psychiatric Association; Washington, DC: 2001b. p. 1-60.

Solovay MR, Shenton ME, Holzman PS. Comparative studies of thought disorder: I. Mania and schizophrenia. Arch. Gen. Psychiatry 1987;44:13-20. [PubMed: 3800579]

Spitzer, RL.; Williams, JBW.; Gibbon, M.; First, MB. Structured Clinical Interview of DSM-III-R-Patient Edition. American Psychiatric Press; Washington, DC: 1990.

Staal WG, Hulshoff Pol HE, Schnack HG, Hoogendoorn MLC, Jellema K, Kahn RS. Structural brain abnormalities in patients with schizophrenia and their healthy siblings. Am. J. Psychiatry 2000;157:416-421. [PubMed: 10698818]

Sullivan EV, Mathalon DH, Lim KO, Marsh L, Pfefferbaum A. Patterns of regional cortical dysmorphology distinguishing schizophrenia and chronic alcoholism. Biol. Psychiatry 1998;43:118131. [PubMed: 9474444]

Swayze VW II, Andreasen NC, Alliger RJ, Yuh WTC, Ehrhardt JC. Subcortical and temporal structures in affective disorder and schizophrenia: a magnetic resonance imaging study. Biol. Psychiatry 1992;31:221-240. [PubMed: 1547297]

Tune L, Barta P, Wong D, Powers RE, Pearlson G, Tien AY, et al. Striatal dopamine D2 receptor quantification and superior temporal gyrus: volume determination in 14 chronic schizophrenic subjects. Psychiatry Res 1996;67:155-158. [PubMed: 8876015]

Vita A, Dieci M, Giobbio GM, Caputo A, Ghiringhelli L, Comazzi M, et al. Language and thought disorder in schizophrenia: brain morphological correlates. Schizophr. Res 1995;15:243-251. [PubMed: 7632621]

Wechsler, D. Wechsler Adult Intelligence Scale—Revised. Harcourt-Brace-Jovanovich; New York, NY: 1981.

Wible, CG.; Shenton, ME.; McCarley, RW. Functional neuroanatomy of the limbic system and the planum temporale. In: Rama Krishnan, KR.; Doraiswamy, PM., editors. Brain Imaging in Clinical Psychiatry. Marcel Dekker; New York: 1997. p. 63-101.

Wise RJS, Scott SK, Blank SC, Mummery CJ, Murphy K, Warburton EA. Separate neural subsystems within 'Wernicke's area'. Brain 2001;124:83-95. [PubMed: 11133789]

Woodruff PW, Wright IC, Shuriquie N, Russouw H, Rushe T, Howard RJ, et al. Structural brain abnormalities in male schizophrenics reflect fronto-temporal dissociation. Psych. Med 1997;27:1257-1266.

Zipursky RB, Marsh L, Lim KO, DeMent S, Shear PK, Sullivan EV, et al. Volumetric MRI assessment of temporal lobe structures in schizophrenia. Biol. Psychiatry 1994;35:501-516. [PubMed: 8038294] 


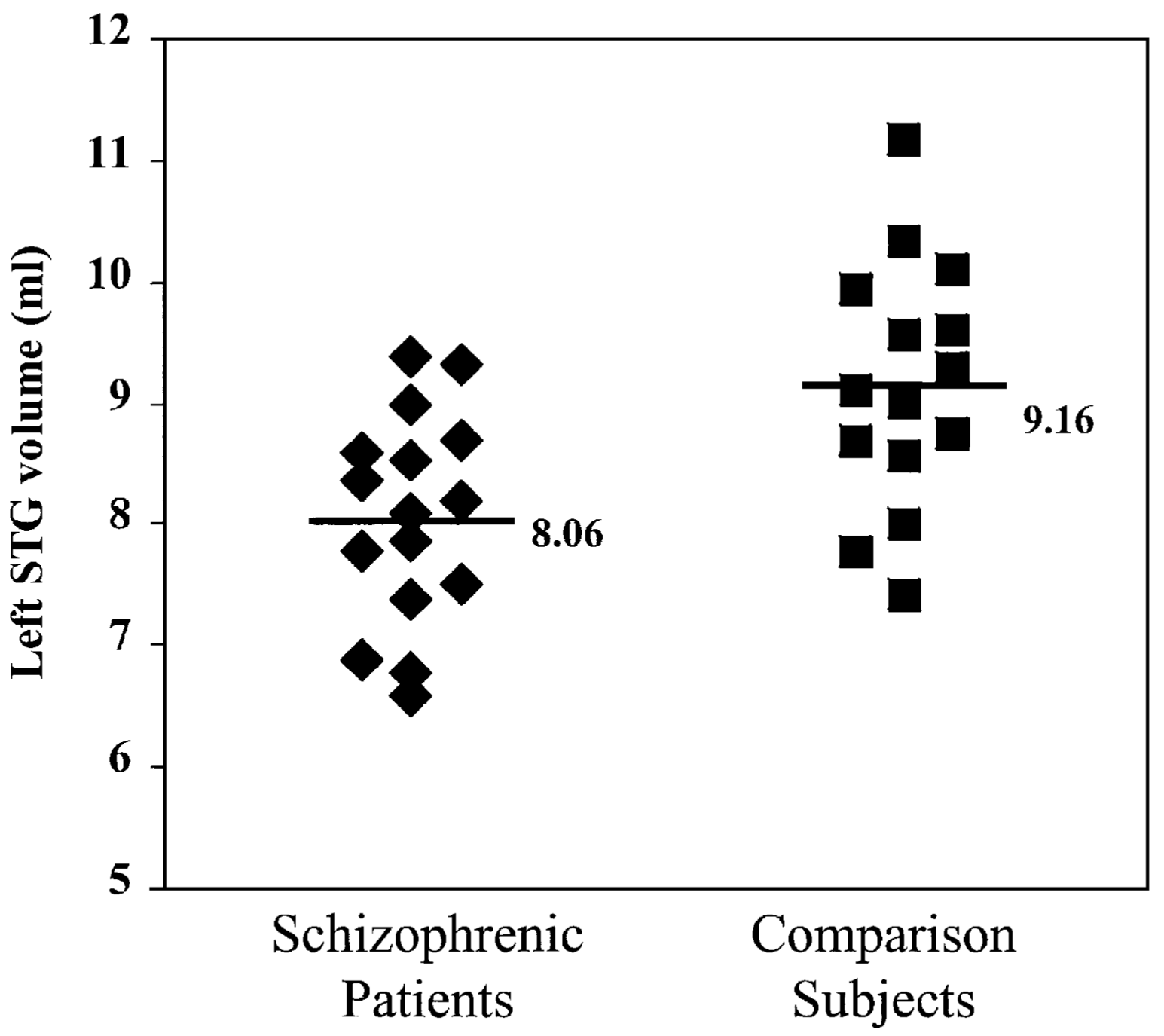

Fig. 1.

Absolute gray matter volume of the left superior temporal gyrus for subjects in each group. Horizontal lines and values indicate group means. $N=16$ for schizophrenia group; $N=15$ for normal comparison group. 


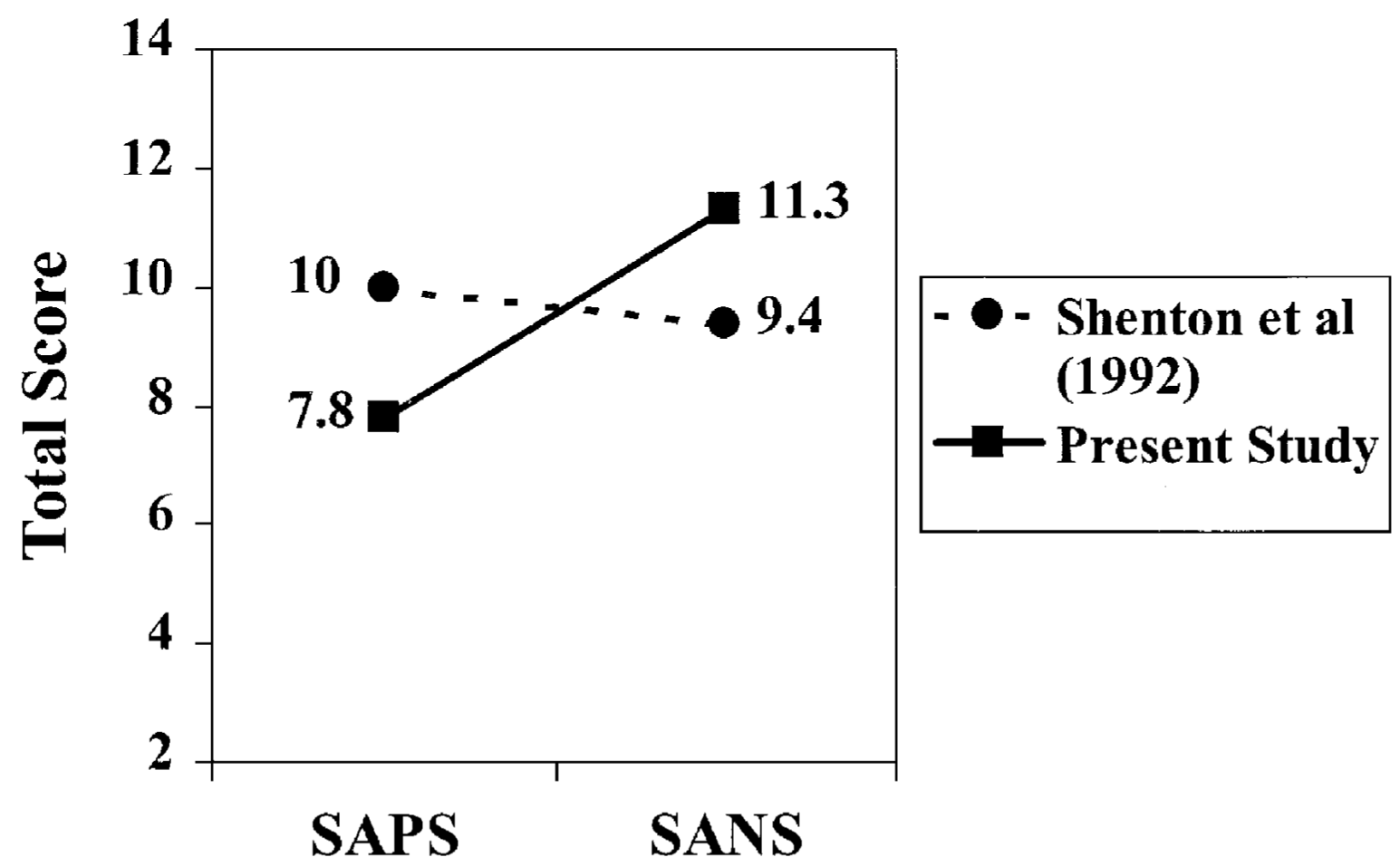

Fig. 2.

Means for total SAPS and SANS scores from the Shenton et al. (1992) study and the present study, depicting greater total SANS scores of the subjects in the present study and greater total SAPS scores of subjects in the Shenton et al. (1992) study [interaction of score and group: $F$ $(1,28)=4.761, p<0.038]$. 


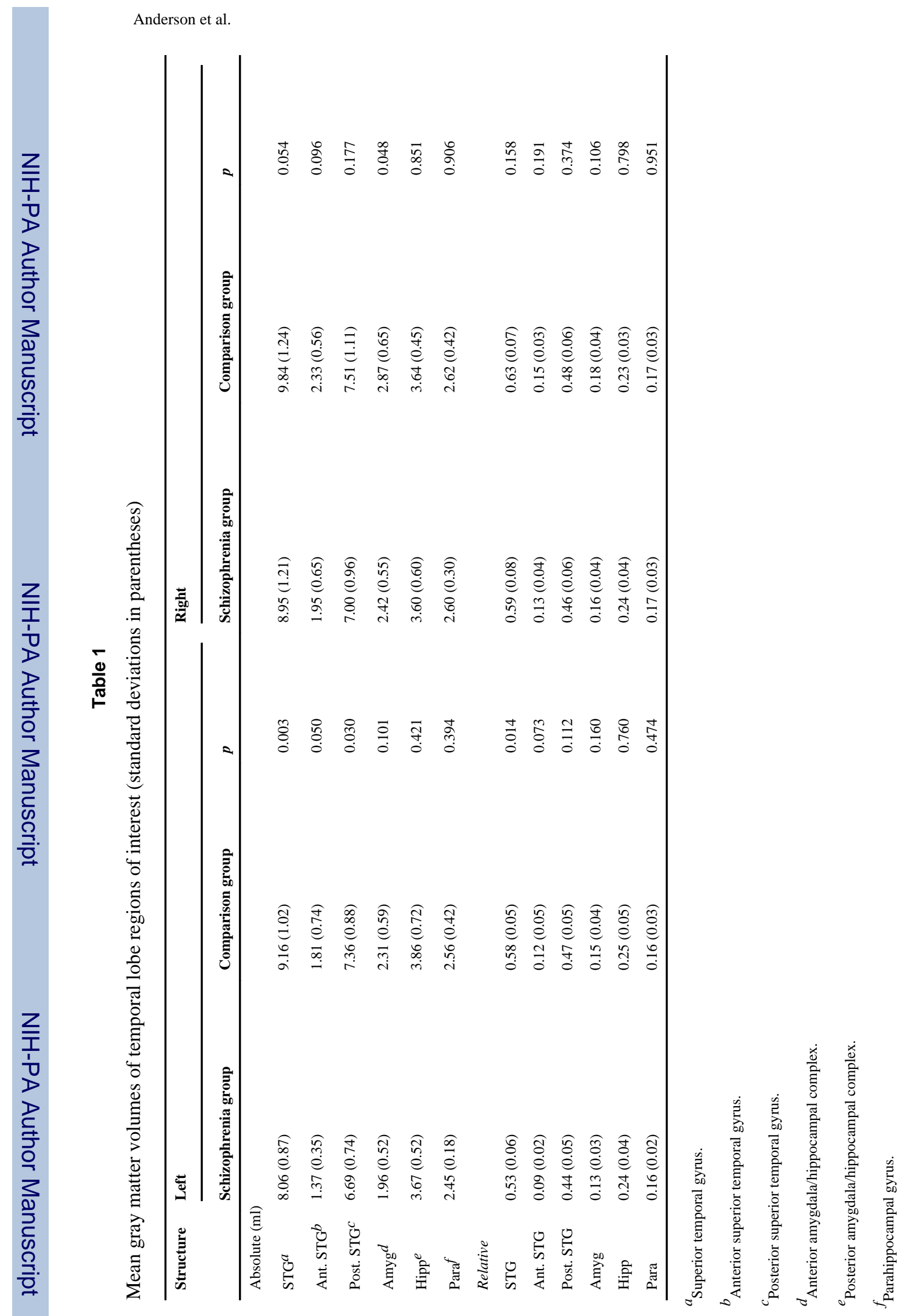

Schizophr Res. Author manuscript; available in PMC 2010 March 25. 